\title{
Visceral Fat Accumulation Is Associated with Increased Mortality Rate after Transcatheter Arterial Chemoembolization in Patients with Hepatocellular Carcinoma
}

\author{
Masaya Saito ${ }^{*}$, Yoshihiko Yano1,2, Hirotaka Hirano1, Kenji Momose1, \\ Yuki Kawano1, Masaru Yoshida1,3, Takeshi Azuma1 \\ ${ }^{1}$ Division of Gastroenterology, Department of Internal Medicine, Graduate School of Medicine, \\ Kobe University, Kobe, Japan \\ ${ }^{2}$ Center for Infectious Diseases, Graduate School of Medicine, Kobe University, Kobe, Japan \\ ${ }^{3}$ Division of Metabolomics Research, Department of Internal Medicine, Graduate School of Medicine, \\ Kobe University, Kobe, Japan \\ Email: "masaya@med.kobe-u.ac.jp
}

Received 24 September 2015; accepted 30 November 2015; published 3 December 2015

Copyright $@ 2015$ by authors and Scientific Research Publishing Inc.

This work is licensed under the Creative Commons Attribution International License (CC BY). http://creativecommons.org/licenses/by/4.0/

(c) () Open Access

\section{Abstract}

Aim: Transcatheter arterial chemoembolization (TACE) is thought to be a safe and effective treatment for hepatocellular carcinoma (HCC). However, in some HCC patients, it potentially shortens survival due to liver damage. We aimed to identify independent factors to predict overall survival of HCC after TACE. Methods: We included a total of 96 consecutive HCC patients who underwent TACE at Kobe University Hospital. Areas of skeletal muscle and fat tissue were measured by computed tomography (CT) scan before TACE. We divided the patients into two groups in terms of the presence or absence of 1-year mortality after TACE. Factors associated with 1-year mortality after TACE were assessed by multivariate analyses, and the optimal cut-off values were evaluated using a propensity score. Results: Multivariate analyses showed that visceral fat accumulation on CT was an independent factor associated with 1 -year mortality after TACE $(p=0.033)$. There were no differences in skeletal muscle area and subcutaneous and intermuscular fat area between the two groups. Cut-off values for visceral fat area associated with 1-year mortality after TACE were defined as $33.3 \mathrm{~cm}^{2} / \mathrm{m}^{2}$ for males and $24.4 \mathrm{~cm}^{2} / \mathrm{m}^{2}$ for females. Conclusions: High visceral fat area was a prognostic factor associated with increased mortality rate in HCC patients undergoing TACE. Using this value, 1-year mortality risk after TACE would be better estimated before the day TACE was performed.

\footnotetext{
*Corresponding author.
}

How to cite this paper: Saito, M., Yano, Y., Hirano, H., Momose, K., Kawano, Y., Yoshida, M. and Azuma, T. (2015) Visceral Fat Accumulation Is Associated with Increased Mortality Rate after Transcatheter Arterial Chemoembolization in Patients with Hepatocellular Carcinoma. Journal of Cancer Therapy, 6, 1124-1136. http://dx.doi.org/10.4236/jct.2015.613122 


\section{Keywords}

\section{Glucose Metabolism, Liver Cirrhosis, Obesity, Overall Survival, Sarcopenia}

\section{Introduction}

Transcatheter arterial chemoembolization (TACE) is the most widely used first-line treatment in the western world and Asia for patients with unresectable hepatocellular carcinoma (HCC). TACE displays a tumor-response rate of 30\% - 60\% [1]-[3] and shows a survival benefit compared with that in controls [4]-[6]. However, TACE against HCC sometimes worsens liver parenchymal function. The deterioration of liver function due to ischemia of the non-tumoral liver following TACE can occasionally lead to the shortening of life expectancy [7]-[9]. Current guidelines for HCC management recommend mortality risk estimates as a decision-making support [10].

Several staging systems before HCC treatment have been developed for mortality risk estimates. The best known systems are Child's score [11], Model for End-stage Liver Disease (MELD) score [12], and Tumor Node Metastasis (TNM) stage [13]. Moreover, previous studies showed that obesity influenced the prognosis of HCC [14] [15]. In addition, we previously reported that energy metabolism could be associated with overall survival in HCC patients after TACE [16]. Visceral fat is a metabolically active component, and is reported to be a prognostic marker of other tumors [17]. Visceral fat can be measured by computed tomography (CT) scan [18], which is routinely used to screen for HCC lesions in cirrhotic patients, and provides precise, objective, and reproducible quantification of visceral fat mass.

In the present study, we aimed to identify independent factors to predict overall survival of HCC after TACE. The pre-therapeutic prediction of overall survival after TACE would help in improving awareness of the development of jaundice, as cites and encephalopathy. To our knowledge, there have been no studies that examine whether visceral fat determined by CT scan can become a new prognostic model after any HCC treatment.

\section{Patients and Methods}

\subsection{Ethics Statement}

All patients provided written consent to participate in the study, and the protocol was approved by the institutional ethics review board at Kobe University. This study complies with the standards of the Declaration of Helsinki and current ethical guidelines.

\subsection{Patients}

We included a total of 96 consecutive patients (mean age: 70.2 years, range: 41 - 87 years; male: female ratio: 61:35) who underwent TACE for HCC between July 2009 and October 2010 at Kobe University Hospital. All patients were Japanese and had liver cirrhosis that had been diagnosed on the basis of laboratory data, ultrasonography, and CT scan. According to the modified Child's classification [11], 52 patients were classified into Child's grade A and 44 were classified into grade B. The etiology of cirrhosis was as follows: hepatitis B in 12 patients, hepatitis $C$ in 66 , alcoholic liver dysfunction in 31 , primary biliary cirrhosis in 2 , autoimmune hepatitis in 1 , non-alcoholic fatty liver disease (NAFLD) in 4 , and unknown in 1, although each category overlaps with others (Table 1). The diagnosis of HCC was based on findings obtained from contrast-enhanced X-ray CT scans. As cites was confirmed by ultrasonography and CT.

In Japan, a consensus-based clinical practice manual proposed by the Japan Society of Hepatology is widely used for the management of HCC [19]. Those who had 4 or more lesions, those who had 3 or fewer lesions and tumor of $3 \mathrm{~cm}$ or more in size, and those who had mild portal invasion without an extra-hepatic lesion were eligible for this study. In addition, those who did not consent to a liver resection, difficult cases in terms of undertaking radiofrequency ablation (RFA) and percutaneous ethanol injection (PEI), and those who were elderly, that is, over 80 years old, were also eligible. Those who had refractory as cites, overt encephalopathy, uncontrolled esophageal varices, severe jaundice, Child's grade C, portal vein trunk thrombosis, or poor performance status (PS) of more than 2 were excluded from the study. In addition, those who used intravenous albumin regu- 
Table 1. The background clinical characteristics of the 96 HCC patients who underwent TACE.

\begin{tabular}{|c|c|}
\hline Age (years) & $70.2 \pm 9.2(41-87)$ \\
\hline Sex (male/female) & $61 / 35$ \\
\hline Child's grade A/B & $52 / 44$ \\
\hline \multicolumn{2}{|l|}{ Cause of liver cirrhosis } \\
\hline Hepatitis B & 12 \\
\hline Hepatitis C & 66 \\
\hline Alcoholic liver dysfunction & 31 \\
\hline Primary biliary cirrhosis & 2 \\
\hline Autoimmune hepatitis & 1 \\
\hline Non-alcoholic fatty liver disease & 4 \\
\hline Unknown & 1 \\
\hline
\end{tabular}

Data are n or mean \pm SD (range). Each category overlaps with others.

larly, those who were given a branched-chain amino acid preparation, and those who had dialysis were also excluded. Liver function of these cases was limited to Child's grade A or B.

The follow-up time was defined as the number of months from TACE to last contact with the patient or death. All patients were periodically seen as outpatients at our hospital. The follow-up protocol included clinical assessment by physical examination and biochemistry every month and the use of ultrasonography or contrast-enhanced CT scan every 3 months. In our study, TACE-related mortality was defined as death from a complication within 2 weeks of each session of TACE.

\subsection{Clinical Evaluation}

The clinical evaluation before treatment was performed on the basis of the results of physical examination and laboratory measurements. All patients were analyzed by the Child's score, MELD score, and TNM stage. The duration (days) of febrile episodes (over $37^{\circ} \mathrm{C}$ ) after TACE was recorded, and the therapeutic volume $\left(\mathrm{cm}^{3}\right)$ in TACE was evaluated by observing Lipiodol deposits on a liver CT scan following TACE. Past TACE treatment frequency (number of times) and other treatments (surgical resection, RFA, and PEI) were also documented. Laboratory measurements including prothrombin time, aspartate aminotransferase, alanine aminotransferase, $\gamma$ glutamyltranspeptidase, total bilirubin, cholinesterase, albumin (Alb), prealbumin (preAlb), the branched-chain amino acid/tyrosine ratio, C-reactive protein (CRP), creatinine, fasting plasma glucose, immunoreactive insulin, homeostasis model assessment of insulin resistance, hemoglobin A1c (HbA1c), the indocyanine green dye clearance test (ICG test) retention rate at 15 min, type 4 collagen 7S, hyaluronic acid, alpha fetoprotein (AFP), and des- $\gamma$-carboxy prothrombin (DCP) were assessed before TACE.

\subsection{Protocol for TACE}

TACE for HCC was performed by catheterization via the femoral artery under local anesthesia, with super-selective catheterization of the hepatic artery feeding the tumor, unless bilobar tumors were involved, in which case chemoembolization was performed in the appropriate hepatic artery. Depending on the tumor size, various amounts of an emulsion of $20 \mathrm{mg}$ of Farmorubicin (epirubicin hydrochloride; Pfizer, USA) and $3 \mathrm{ml}$ of Lipiodol (the iodine addition products of the ethyl esters of fatty acids obtained from poppy seed oil; Mitsui, Japan) at a 1:1 volume ratio were injected under fluoroscopic monitoring. This was followed by embolization with gelatin (Gelpart; Yamanouchi, Japan). Following TACE, a liver CT scan was performed to calculate the therapeutic volume from the distribution of Lipiodol deposits.

\subsection{CT Analyses of Body Composition Variables}

CT scans used for analyses were performed solely for the purpose of diagnosing and staging HCC. A transverse 
CT image from L3 in the inferior direction was assessed from each scan. Images were analyzed with Slice Omantic V5.0 software (Tomo Vision, Montreal, Quebec, Canada), which enables specific tissue demarcation by using previously reported Hounsfield unit (HU) thresholds. Skeletal muscle is identified and qualified by HU thresholds of -29 to +150 [20]. Muscles in the L3 region encompass psoas, erector, spinae, quadratus lumborum, transversus abdominis, external and internal obliques, and rectus abdominis. The following HU thresholds were used for fat tissues: -190 to -30 for subcutaneous and intermuscular fat tissues [21], and -150 to -50 for visceral fat tissues [22]. With these specific HU thresholds, measurements of the skeletal muscle are not influenced by the presence of ascites in patients with cirrhosis. Cross-sectional areas $\left(\mathrm{cm}^{2}\right)$ were automatically computed by summing tissue pixels and multiplying by pixel surface area. All CT images were analyzed by two trained observers. Cross-sectional area of muscle and fat tissue was normalized for stature $\left(\mathrm{cm}^{2} / \mathrm{m}^{2}\right)$, as reported elsewhere [23]. The L3 skeletal muscle was expressed as cross-sectional muscle area/height, and cut-offs for sarcopenia were based on a CT-based sarcopenic study (L3 skeletal muscle area/ height, $\leq 38.5 \mathrm{~cm}^{2} / \mathrm{m}^{2}$ for females and $\leq 52.4 \mathrm{~cm}^{2} / \mathrm{m}^{2}$ for males) [24] [25].

\subsection{Statistical Analysis}

Statistical analyses were conducted using SPSS Statistics 18.0 (SPSS Inc., Chicago, IL, USA). Univariate analyses of demographic factors were performed to investigate the difference between two groups. Multivariate analyses were constructed using backward stepwise logistic regression. The predictive ability of the model was quantified using each significant multivariate factor and 1-year mortality (area under ROC, AUC). An optimal cut-off point was a propensity score on the maximal sum of sensitivity and specificity. The relationship between two variables was investigated by Spearman's correlation coefficient test. Log-rank test was performed to compare the survival distributions. Values of $p<0.05$ were considered significant.

\section{Results}

\subsection{Factor Analyses Associated with 1-Year Mortality after TACE}

One-year mortality after TACE was found in 32 patients out of 96 (1-year mortality group), and 1-year survival was detected in 64 patients (1-year survival group). Table 2 shows the results of univariate analyses comparing several factors before TACE between the two groups. Body mass index (BMI), Child's score, TNM stage, past treatment frequency of TACE, visceral fat area, CRP, ICG test retention rate at $15 \mathrm{~min}$, AFP, and DCP were significantly higher in the 1-year mortality group than in the survival group $(p=0.002,0.009,0.001,0.000$, $0.016,0.023,0.005,0.004$, and 0.000 , respectively). Alb and preAlb were significantly lower in the 1-year mortality group than in the survival group ( $p=0.016$ and 0.044 , respectively). Skeletal muscle area and subcutaneous and intermuscular fat area showed no significant differences between the two groups $(p=0.051$ and 0.105 , respectively). Table 3 shows the results of univariate analyses comparing several factors after TACE between the two groups. Therapeutic volume and febrile duration were significantly higher in the 1-year mortality group than in the survival group ( $p=0.001$ and 0.000 , respectively).

We found a significant positive correlation between visceral fat area and BMI ( $p=0.000, r=0.423$ ) (Figure 1). Multicollinearity was also detected between Alb and preAlb, so BMI and preAlb were not introduced into the multivariate logistic regression analyses. The 9 significant univariate factors other than them were introduced into a multivariable logistic model. Backward stepwise multivariate regression analyses showed that visceral fat, ICG test retention rate at $15 \mathrm{~min}$, DCP, and past treatment frequency of TACE were independent factors associated with 1 -year mortality after TACE ( $p=0.033,0.001,0.009$, and 0.007 , respectively) (Table 4 ).

\subsection{Evaluation of Cut-Off Values of Visceral Fat Area}

The median visceral fat area of all patients was $34.3 \mathrm{~cm}^{2} / \mathrm{m}^{2}$ (range $2.3-111.3 \mathrm{~cm}^{2} / \mathrm{m}^{2}$ ) and, in male patients, it was $35.9 \mathrm{~cm}^{2} / \mathrm{m}^{2}$ (range $2.3-111.3 \mathrm{~cm}^{2} / \mathrm{m}^{2}$ ), which was greater than that in female patients (median $26.1 \mathrm{~cm}^{2} / \mathrm{m}^{2}$, range $4.9-69.8 \mathrm{~cm}^{2} / \mathrm{m}^{2} ; p=0.029$ ) (Figure 2). Using propensity score, cut-off values for visceral fat area associated with 1-year mortality after TACE were defined as $33.3 \mathrm{~cm}^{2} / \mathrm{m}^{2}$ for males and $24.4 \mathrm{~cm}^{2} / \mathrm{m}^{2}$ for females, which is recognized as a measure of metabolic abnormalities in Japan [26]. We divided the study population into two groups: high visceral fat area (H-VFA) or low visceral fat area (L-VFA). 
Table 2. Univariate analyses comparing several factors (before TACE) between 1-year mortality group and survival group (n =96).

\begin{tabular}{|c|c|c|c|c|c|}
\hline \multirow{3}{*}{$\begin{array}{c}\text { Factor } \\
\text { (before TACE) } \\
\text { Age (years) }\end{array}$} & \multirow{2}{*}{\multicolumn{2}{|c|}{$\begin{array}{l}\text { 1-year mortality group } \\
\qquad \mathrm{n}=32\end{array}$}} & \multirow{2}{*}{\multicolumn{2}{|c|}{$\begin{array}{l}\text { 1-year survival group } \\
\qquad \mathrm{n}=64\end{array}$}} & \multirow{3}{*}{$\begin{array}{l}p \text { value } \\
0.669^{\dagger}\end{array}$} \\
\hline & & & & & \\
\hline & 71 & $(56-86)$ & 71 & $(41-87)$ & \\
\hline Sex (male/female) & \multicolumn{2}{|c|}{$20 / 12$} & \multicolumn{2}{|c|}{$41 / 23$} & $0.881^{\S}$ \\
\hline Height (cm) & $158.4 \pm 8$ & $(144.0-174.5)$ & $161.0 \pm 9.3$ & $(138.5-179.0)$ & $0.088^{*}$ \\
\hline Body weight (kg) & 62.1 & $(48.0-91.5)$ & 59.8 & $(37.0-93.6)$ & $0.280^{\dagger}$ \\
\hline $\operatorname{BMI}\left(\mathrm{kg} / \mathrm{m}^{2}\right)$ & $24.8 \pm 2.7$ & $(19.0-30.0)$ & $22.9 \pm 2.8$ & $(15.6-30.2)$ & $0.002^{*}$ \\
\hline HBs Ag $(+/-)$ & \multicolumn{2}{|c|}{$4 / 28$} & \multicolumn{2}{|c|}{$8 / 56$} & $1.000^{\pi}$ \\
\hline HCV Ab (+/-) & \multicolumn{2}{|c|}{$22 / 10$} & \multicolumn{2}{|c|}{$44 / 20$} & $1.000^{\S}$ \\
\hline Alcohol (over 20 g/day) (+/-) & \multicolumn{2}{|c|}{$9 / 23$} & \multicolumn{2}{|c|}{$22 / 42$} & $0.537^{\S}$ \\
\hline Child's score $(5,6,7,8,9)$ & \multicolumn{2}{|c|}{$7(5-9)$} & \multicolumn{2}{|c|}{$6(5-9)$} & $0.009^{\dagger}$ \\
\hline MELD score & \multicolumn{2}{|c|}{$7.3(6.0$ - 11.9) } & \multicolumn{2}{|c|}{$7.1(6.0-20.7)$} & $0.465^{\dagger}$ \\
\hline TNM stage of HCC (I, II, III, IVa, IVb) & \multicolumn{2}{|c|}{$0 / 8 / 20 / 2 / 2$} & \multicolumn{2}{|c|}{$8 / 23 / 32 / 1$} & $0.001^{*}$ \\
\hline Past treatment frequency of TACE (times) & \multicolumn{2}{|c|}{$3(0-11)$} & \multicolumn{2}{|c|}{$1(0-10)$} & $\mathbf{0 . 0 0 0}^{\dagger}$ \\
\hline Past treatments other than TACE $(+/-)$ & \multicolumn{2}{|c|}{$15 / 17$} & \multicolumn{2}{|c|}{$25 / 39$} & $0.464^{\S}$ \\
\hline Visceral fat area $\left(\mathrm{cm}^{2} / \mathrm{m}^{2}\right)$ & \multicolumn{2}{|c|}{$39.9(12.2-111.3)$} & \multicolumn{2}{|c|}{$30.7(2.3-93.0)$} & $0.016^{\dagger}$ \\
\hline Subcutaneous and intermuscular fat area $\left(\mathrm{cm}^{2} / \mathrm{m}^{2}\right)$ & \multicolumn{2}{|c|}{$57.9 \pm 20.9(7.9-96.8)$} & \multicolumn{2}{|c|}{$51.6 \pm 24.2(2.8-101.0)$} & $0.105^{*}$ \\
\hline Skeletal muscle area $\left(\mathrm{cm}^{2} / \mathrm{m}^{2}\right)$ & \multicolumn{2}{|c|}{$44.5 \pm 6.9(27.1-57.6)$} & $41.6 \pm \varepsilon$ & $(20.5-70.8)$ & $0.051^{*}$ \\
\hline PT (\%) & 77.1 & $(67.1-100.0)$ & 83.2 & $(57.6-100.0)$ & $0.133^{\dagger}$ \\
\hline AST (IU/L) & 47 & $(19-165)$ & 49 & $(18-141)$ & $0.920^{\dagger}$ \\
\hline ALT (IU/L) & 35 & $(11-111)$ & 37 & $(13-130)$ & $0.388^{\dagger}$ \\
\hline$\gamma$-GTP (IU/L) & 52 & $(10-857)$ & 49 & $(13-1188)$ & $0.594^{\dagger}$ \\
\hline T-Bil (mg/dL) & 0.9 & $(0.4-2.5)$ & 0.9 & $(0.3-2.1)$ & $0.935^{\dagger}$ \\
\hline $\mathrm{ChE}(\mathrm{IU} / \mathrm{L})$ & 130 & $(81-348)$ & 168 & $(47-327)$ & $0.062^{\dagger}$ \\
\hline $\operatorname{Alb}(g / d L)$ & 3.2 & $(2.4-4.3)$ & 3.7 & $(2.4-4.8)$ & $\mathbf{0 . 0 1 6}^{\dagger}$ \\
\hline Prealbumin (mg/dL) & 9.6 & $(4.5-28.2)$ & 12.4 & $(4.2-33.1)$ & $0.044^{\dagger}$ \\
\hline BTR & 3.4 & $(1.7-7.3)$ & 4.0 & $(1.5-8.0)$ & $0.076^{\dagger}$ \\
\hline CRP & 0.1 & $(0.1-3.5)$ & 0.1 & $(0.1-6.1)$ & $0.023^{\dagger}$ \\
\hline Crn & 0.77 & $(0.46-1.93)$ & 0.75 & $(0.44-4.91)$ & $0.616^{\dagger}$ \\
\hline FPG (mg/dL) & 100 & $(71-207)$ & 101 & $(74-351)$ & $0.834^{\dagger}$ \\
\hline IRI $(\mu \mathrm{U} / \mathrm{mL})$ & 14 & $(4-40)$ & 11 & $(1-84)$ & $0.243^{\dagger}$ \\
\hline HOMA-IR & 3.01 & $(0.81-12.10)$ & 2.58 & $(0.19-43.30)$ & $0.310^{\dagger}$ \\
\hline HbA1c (\%) & 5.3 & $(4.4-6.9)$ & 5.4 & $(4.3-10.2)$ & $0.112^{\dagger}$ \\
\hline ICG test retention rate at $15 \mathrm{~min}(\%)$ & 31.6 & $(8.5-75.6)$ & 21.9 & $(3.5-59.5)$ & $0.005^{\dagger}$ \\
\hline Type 4 collagen 7S (ng/mL) & 7.3 & $(5.0-11.0)$ & 6.5 & $(3.0-16.0)$ & $0.091^{\dagger}$ \\
\hline $\mathrm{HA}(\mathrm{ng} / \mathrm{mL})$ & 286.9 & $(53.8-1483.6)$ & 299 & $(12.4-1237.8)$ & $0.841^{\dagger}$ \\
\hline AFP (ng/mL) & 80 & $(6-94260)$ & 17 & $(2-14183)$ & $\mathbf{0 . 0 0 4}^{\dagger}$ \\
\hline DCP & 1249 & $(16-688500)$ & 90 & $(14-7138)$ & $\mathbf{0 . 0 0 0}^{\dagger}$ \\
\hline
\end{tabular}

Data represent $\mathrm{n}$, mean $\pm \mathrm{SD}$ (range), or median (range). The data were evaluated with the ${ }^{\S}$ Chi-square test, ${ }^{*}$ two-sample t-test, ${ }^{\dagger}$ Wilcoxon rank sum test, or "Fisher's exact test as appropriate. 


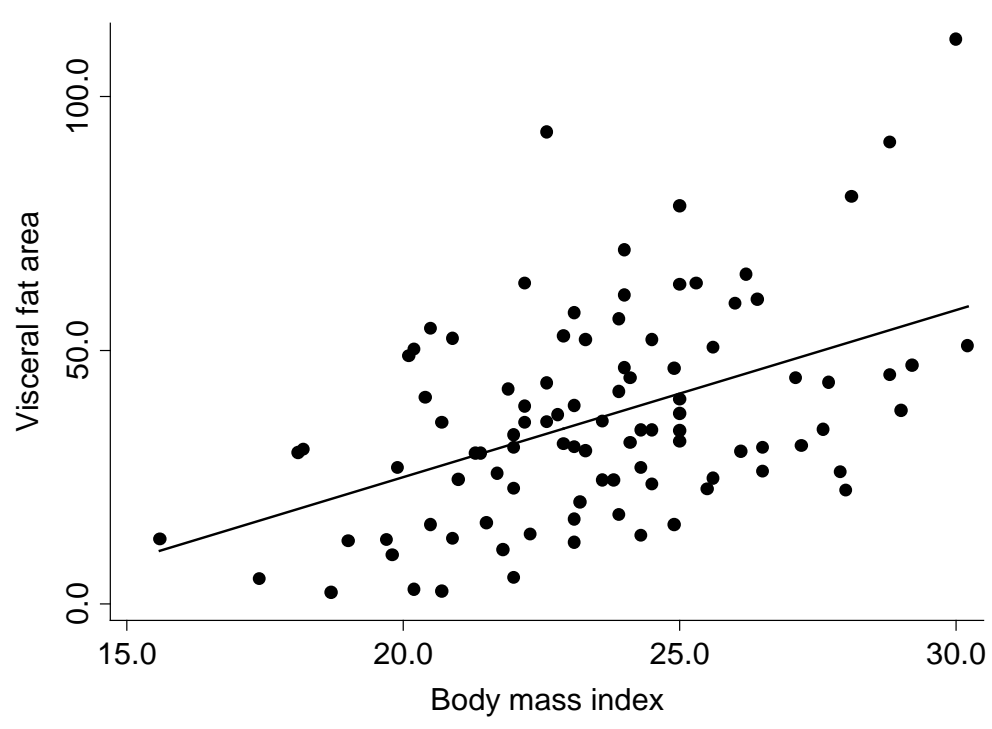

Figure 1. The correlation between visceral fat area and body mass index (BMI) in hepatocellular carcinoma (HCC) patients undergoing transcatheter arterial chemoembolization (TACE). Before TACE, visceral fat area was positively related to BMI by Spearman's correlation coefficient test $(p=0.000, r=0.423)$.

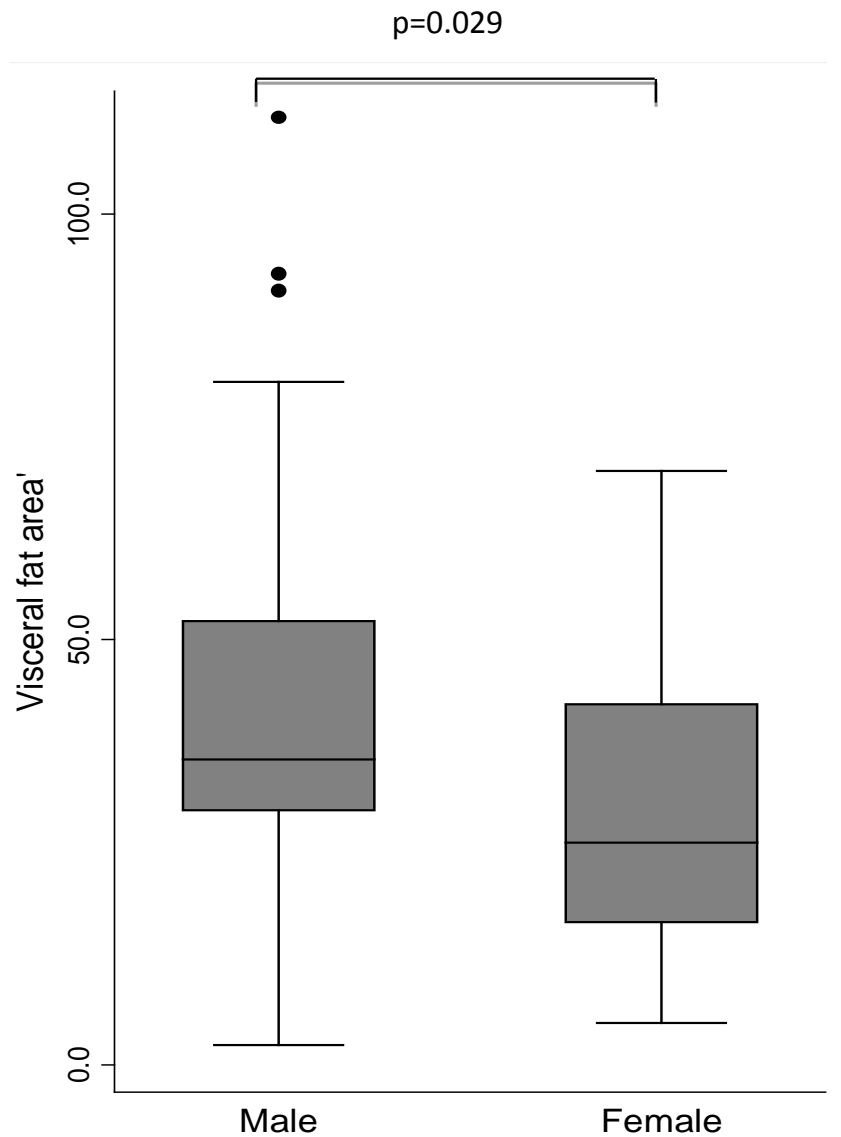

Figure 2. Comparison of visceral fat area between males and females. The median visceral fat area of all patients was $34.3 \mathrm{~cm}^{2} / \mathrm{m}^{2}$ (range $2.3-111.3 \mathrm{~cm}^{2} / \mathrm{m}^{2}$ ); in male patients, it was 35.9 $\mathrm{cm}^{2} / \mathrm{m}^{2}$ (range $2.3-111.3 \mathrm{~cm}^{2} / \mathrm{m}^{2}$ ), which was more than that in female patients (median 26.1 $\mathrm{cm}^{2} / \mathrm{m}^{2}$, range $\left.4.9-69.8 \mathrm{~cm}^{2} / \mathrm{m}^{2} ; p=0.029\right)$. 
Table 3. Univariate analyses comparing several factors (after TACE) between 1-year mortality group and survival group (n $=96)$.

\begin{tabular}{cccc}
\hline Factor & 1-year mortality group & 1-year survival group & $p$ value \\
\hline (after TACE) & $\mathrm{n}=32$ & $\mathrm{n}=64$ & $\mathbf{0 . 0 0 1}^{\dagger}$ \\
\hline Therapeutic volume $\left(\mathrm{cm}^{3}\right)$ & $110.1(11.9-527.2)$ & $50.7(2.0-291.4)$ & $\mathbf{0 . 0 0 0}^{\dagger}$ \\
\hline Febrile duration (days) & $7(1-7)$ & $3(0-7)$ & \\
\hline
\end{tabular}

Data represent median (range). The data were evaluated with the ${ }^{\dagger}$ Wilcoxon rank sum test.

Table 4. Multivariate logistic regression analyses comparing the significant univariate factors associated with 1-year mortality after TACE $(\mathrm{n}=96)$.

\begin{tabular}{|c|c|c|c|c|c|c|}
\hline \multirow{2}{*}{ Factor } & \multirow{2}{*}{ B } & \multirow{2}{*}{$\mathrm{SE}$} & \multirow{2}{*}{$p$ value } & \multirow{2}{*}{$\operatorname{Exp}(B)$} & \multicolumn{2}{|c|}{ 95\% CI of EXP(B) } \\
\hline & & & & & Upper limit & Lower limit \\
\hline Visceral fat area & 2.13 & 0.005 & 0.033 & 1.012 & 1.001 & 1.022 \\
\hline ICG test retention rate at $15 \mathrm{~min}(\%)$ & 3.22 & 0.033 & 0.001 & 1.102 & 1.039 & 1.169 \\
\hline DCP & 2.63 & 0.000 & 0.009 & 1.001 & 1.000 & 1.001 \\
\hline Past treatment frequency of TACE & 2.70 & 0.196 & 0.007 & 1.442 & 1.106 & 1.882 \\
\hline
\end{tabular}

B: regression coefficient; CI: confidence interval; $\operatorname{Exp(B):~odds~ratio;~SE:~standard~error.~Multicollinearity~was~detected~between~visceral~fat~area~and~}$ BMI and between Alb and preAlb, so BMI and preAlb were not introduced into the multivariate logistic regression analyses.

\subsection{Factor Analyses Associated with Visceral Fat Area}

H-VFA was found in 58 out of 96 patients (H-VFA group), and L-VFA was detected in 38 patients (L-VFA group). Table 5 shows the results of univariate analyses comparing several factors before TACE between the two groups. Body weight, BMI, TNM stage, subcutaneous and intermuscular fat area, and HbA1c were significantly higher in the H-VFA group than in the L-VFA group $(p=0.003,0.000,0.003,0.001$, and 0.017 , respectively). T-Bil was significantly lower in the H-VFA group than in the L-VFA group $(p=0.044)$. Skeletal muscle area and presence or absence of sarcopenia did not differ between the two groups ( $p=0.232$ and 0.904 , respectively). Table 6 shows the results of univariate analyses comparing several factors after TACE between the two groups. One-year mortality after TACE was significantly higher in the H-VFA group than in the L-VFA group ( $p=0.003$ ).Before the analyses, this study showed that visceral fat area had significantly positive relationships to BMI and 1-year mortality after TACE. Multicollinearity was also detected between body weight and BMI, so they were not introduced into the multivariate logistic regression analyses. The 4 significant univariate factors other than them were introduced into a multivariable logistic model. Backward stepwise multivariate regression analyses showed that TNM stage, subcutaneous and intermuscular fat area, and HbA1c were independent factors associated with visceral fat area ( $p=0.007,0.001$, and 0.028 , respectively) (Table 7).

\subsection{Follow-Up}

All of the 96 patients were completely followed up. During the follow-up, 56 patients died, whereas 40 patients were alive at the end of the study. Out of the 56 dead patients, 36 patients died of liver failure and the other 20 patients died of HCC progression. TACE- and/or sepsis-related mortality was not observed in this study. The median follow-up period was 24 months ( 2 - 41 months). The median survival time was 27.0 months (95\% CI 16.146 - 31.854). The overall survival rates were $66.7 \%$ and $49.0 \%$ at 12 and 24 months, respectively. Using the Kaplan-Meier method, the distribution of the 96 patients between H-VFA and L-VFA associated with 1-year mortality after TACE is described in Figure 3. Overall survival was significantly shorter in the H-VFA group than in the L-VFA group $(p=0.017)$.

\section{Discussion}

Coinciding with the rising incidence of HCC, the prevalence of obesity has increased rapidly over the past two 
Table 5. Univariate analyses comparing several factors (before TACE) between H-VFA group and L-VFA group ( $\mathrm{n}=96$ ).

\begin{tabular}{|c|c|c|c|c|c|}
\hline \multirow{3}{*}{$\begin{array}{c}\text { Factor } \\
\text { (Before TACE) } \\
\text { Age (years) }\end{array}$} & \multirow{2}{*}{\multicolumn{2}{|c|}{$\begin{array}{c}\text { H-VFA group } \\
n=58\end{array}$}} & \multirow{2}{*}{\multicolumn{2}{|c|}{$\begin{array}{c}\text { L-VFA group } \\
\qquad n=38\end{array}$}} & \multirow{3}{*}{$\begin{array}{l}p \text { value } \\
0.784^{\dagger}\end{array}$} \\
\hline & & & & & \\
\hline & 71 & $(41-87)$ & 71 & $(55-83)$ & \\
\hline Sex (male/female) & \multicolumn{2}{|c|}{$37 / 21$} & \multicolumn{2}{|c|}{$24 / 14$} & $0.950^{\S}$ \\
\hline Height (cm) & $160.0 \pm 9.8$ & $(138.5-179.0)$ & $160.2 \pm 7.6$ & $(147.7-176.9)$ & $0.452^{*}$ \\
\hline Body weight (kg) & 63.1 & $(40.2-93.6)$ & 56.8 & $(37.0-73.6)$ & $\mathbf{0 . 0 0 3}^{\dagger}$ \\
\hline BMI $\left(\mathrm{kg} / \mathrm{m}^{2}\right)$ & $24.4 \pm 2.6$ & $(20.1-30.2)$ & $22.2 \pm 2.8$ & $(15.6-28.0)$ & $0.000^{*}$ \\
\hline HBs Ag (+/-) & \multicolumn{2}{|c|}{$7 / 51$} & \multicolumn{2}{|c|}{$5 / 33$} & $1.000^{\pi}$ \\
\hline HCV Ab (+/-) & \multicolumn{2}{|c|}{$36 / 22$} & \multicolumn{2}{|c|}{$30 / 8$} & $0.081^{\S}$ \\
\hline Alcohol (over 20 g/day)(+/-) & \multicolumn{2}{|c|}{$20 / 38$} & \multicolumn{2}{|c|}{$11 / 27$} & $0.571^{\S}$ \\
\hline Child's score $(5,6,7,8,9)$ & \multicolumn{2}{|c|}{$6(5-9)$} & \multicolumn{2}{|c|}{$7(5-9)$} & $0.390^{\dagger}$ \\
\hline MELD score & \multicolumn{2}{|c|}{$7.0(6.0-12.3)$} & \multicolumn{2}{|c|}{$7.3(6.0-20.7)$} & $0.227^{\dagger}$ \\
\hline TNM stage of HCC (I, II, III, IVa, IVb) & \multicolumn{2}{|c|}{$5 / 11 / 37 / 3 / 2$} & \multicolumn{2}{|c|}{$3 / 20 / 15 / 0 / 0$} & $0.003^{*}$ \\
\hline Past treatment frequency of TACE (times) & \multicolumn{2}{|c|}{$1(0-11)$} & \multicolumn{2}{|c|}{$1(0-10)$} & $0.283^{\dagger}$ \\
\hline Past treatments other than TACE $(+/-)$ & \multicolumn{2}{|c|}{$24 / 34$} & \multicolumn{2}{|c|}{$16 / 22$} & $0.944^{\S}$ \\
\hline Subcutaneous and intermuscular fat area $\left(\mathrm{cm}^{2} / \mathrm{m}^{2}\right)$ & \multicolumn{2}{|c|}{$59.8 \pm 18.1(33.6-97.8)$} & \multicolumn{2}{|c|}{$44.4 \pm 27.1(2.8-101.4)$} & $0.001^{*}$ \\
\hline Skeletal muscle area $\left(\mathrm{cm}^{2} / \mathrm{m}^{2}\right)$ & \multicolumn{2}{|c|}{$43.0 \pm 8.7(20.5-70.8)$} & \multicolumn{2}{|c|}{$41.8 \pm 7.2(21.7-57.6)$} & $0.232^{*}$ \\
\hline Sarcopenia (+/-) & \multicolumn{2}{|c|}{$39 / 19$} & & $5 / 12$ & $0.904^{\S}$ \\
\hline PT (\%) & 80.3 & $(58.5-100.0)$ & 82.4 & $(57.6-100.0)$ & $0.827^{\dagger}$ \\
\hline AST (IU/L) & 48 & $(19-165)$ & 49 & $(18-141)$ & $0.412^{\dagger}$ \\
\hline ALT (IU/L) & 36 & $(11-111)$ & 37 & $(13-130)$ & $0.875^{\dagger}$ \\
\hline$\gamma$-GTP (IU/L) & 51 & $(20-857)$ & 47 & $(10-1188)$ & $0.436^{\dagger}$ \\
\hline T-Bil (mg/dL) & 0.8 & $(0.3-2.5)$ & 1.0 & $(0.3-2.5)$ & $\mathbf{0 . 0 4 4}^{\dagger}$ \\
\hline $\mathrm{ChE}(\mathrm{IU} / \mathrm{L})$ & 161 & $(81-348)$ & 145 & $(47-327)$ & $0.123^{\dagger}$ \\
\hline Alb (g/dL) & 3.6 & $(2.4-4.8)$ & 3.4 & $(2.4-4.5)$ & $0.346^{\dagger}$ \\
\hline Prealbumin (mg/dL) & 11.5 & $(4.2-28.2)$ & 10.9 & $(4.5-33.1)$ & $0.479^{\dagger}$ \\
\hline BTR & 3.9 & $(1.5-7.4)$ & 4.0 & $(1.9-8.0)$ & $0.691^{\dagger}$ \\
\hline CRP & 0.1 & $(0.1-6.1)$ & 0.1 & $(0.1-2.9)$ & $0.401^{\dagger}$ \\
\hline Crn & 0.77 & $(0.44-1.93)$ & 0.72 & $(0.45-4.91)$ & $0.514^{\dagger}$ \\
\hline FPG (mg/dL) & 104 & $(71-234)$ & 98 & $(74-351)$ & $0.142^{\dagger}$ \\
\hline IRI $(\mu \mathrm{U} / \mathrm{mL})$ & 11 & $(4-84)$ & 11 & $(1-40)$ & $0.280^{\dagger}$ \\
\hline HOMA-IR & 2.8 & $(0.81-43.30)$ & 2.64 & $(0.19-20.80)$ & $0.310^{\dagger}$ \\
\hline HbA1c (\%) & 5.6 & $(4.4-8.2)$ & 5.1 & $(4.3-10.2)$ & $0.017^{\dagger}$ \\
\hline ICG test retention rate at $15 \mathrm{~min}(\%)$ & 25.4 & $(5.5-59.5)$ & 23.4 & $(3.5-75.6)$ & $0.919^{\dagger}$ \\
\hline Type 4 collagen 7S (ng/mL) & 6.5 & $(3.0-14.0)$ & 7.8 & $(3.6-16.0)$ & $0.132^{\dagger}$ \\
\hline $\mathrm{HA}(\mathrm{ng} / \mathrm{mL})$ & 229 & $(12.4-1483.6)$ & 332.9 & $(48.1$ - 1237.8) & $0.074^{\dagger}$ \\
\hline AFP (ng/mL) & 36 & $(2-94260)$ & 26 & $(5-14183)$ & $0.694^{\dagger}$ \\
\hline DCP & 250 & $(14-688500)$ & 93 & $(14-12749)$ & $0.076^{\dagger}$ \\
\hline
\end{tabular}

Data represent $n$, mean \pm SD (range), or median (range). The data were evaluated with the ${ }^{\S}$ Chi-square test, ${ }^{*}$ two-sample t-test, ${ }^{\dagger}$ Wilcoxon rank sum test, or "Fisher's exact test as appropriate. 


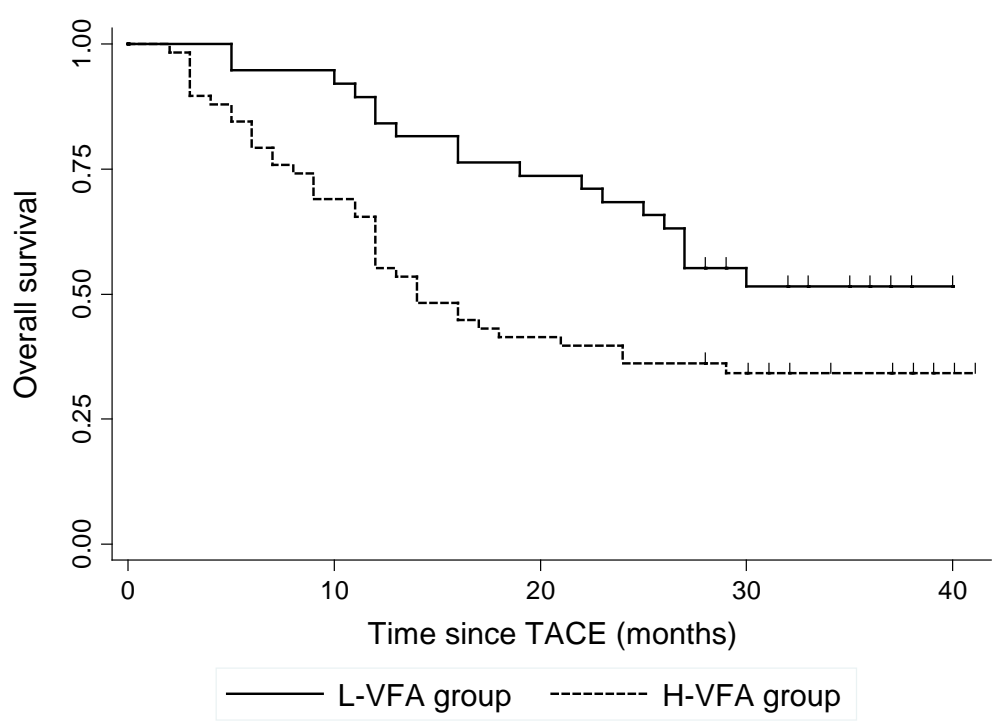

Figure 3. Overall survival according to visceral fat area in 96 patients with HCC after TACE. All of the 96 patients were completely followed up. During follow-up, 56 patients died, whereas 40 patients were alive at the end of the study. TACE-related mortality was not observed in this study. The median follow-up period was 24 months ( 2 - 41 months). The median survival time was 27.0 months (95\% CI 16.146 - 31.854). The overall survival rates were $66.7 \%$ and $49.0 \%$ at 12 and 24 months, respectively. Overall survival was significantly shorter in the high-VFA (H-VFA) group than in the low-VFA (L-VFA) group $(p=0.017)$. The H-VFA group is designated by a dotted line and the L-VFA group by a solid line.

Table 6. Univariate analyses comparing several factors (after TACE) between H-VFA group and L-VFA group ( $\mathrm{n}=96$ ).

\begin{tabular}{cccc}
\hline Factor & H-VFA group & L-VFA group & \\
(After TACE) & $\mathrm{n}=58$ & $\mathrm{n}=38$ & \\
\hline Therapeutic volume $\left(\mathrm{cm}^{3}\right)$ & $78.4(2.0-527.2)$ & $52.1(3.1-316.0)$ & $0.120^{\dagger}$ \\
Febrile duration (days) & $5(0-7)$ & $5(0-7)$ & $0.924^{\dagger}$ \\
1-year mortality after TACE (dead/alive) & $26 / 32$ & $6 / 32$ & $\mathbf{0 . 0 0 3}^{\S}$ \\
\hline
\end{tabular}

Data represent nor median (range). The data were evaluated with the ${ }^{\S}$ Chi-square test or ${ }^{\dagger}$ Wilcoxon rank sum test as appropriate.

Table 7. Multivariate logistic regression analyses comparing the significant univariate factors associated with H-VFA or L-VFA ( $n=96)$.

\begin{tabular}{|c|c|c|c|c|c|c|}
\hline \multirow{2}{*}{ Factor } & \multirow{2}{*}{ B } & \multirow{2}{*}{$\mathrm{SE}$} & \multirow{2}{*}{$p$ value } & \multirow{2}{*}{$\operatorname{Exp}(B)$} & \multicolumn{2}{|c|}{$95 \%$ CI of EXP(B) } \\
\hline & & & & & Upper limit & Lower limit \\
\hline TNM stage & 2.68 & 0.852 & 0.007 & 2.495 & 1.277 & 4.873 \\
\hline Subcutaneous and intermuscular fat area & 3.26 & 0.013 & 0.001 & 1.043 & 1.017 & 1.070 \\
\hline HbA1c & 2.19 & 0.54 & 0.028 & 1.878 & 1.069 & 3.299 \\
\hline
\end{tabular}

B: regression coefficient; CI: confidence interval; Exp (B): odds ratio; SE: standard error. Before the analyses, this study showed that visceral fat area had significantly positive relationships to BMI and 1-year mortality after TACE. Multicollinearity was also detected between body weight and BMI, so they were not introduced into the multivariate logistic regression analyses.

decades [14] [27]-[31]. Obesity is now recognized as one of the most challenging public health issues worldwide. Calle et al. reported that obesity was related to increased mortality from HCC [14]. To elucidate the impact of the type of obesity further, we measured both visceral fat tissue and subcutaneous and intermuscular fat tissue. Our study showed that high visceral fat tissue, but not subcutaneous and intermuscular fat tissue, was an independent factor associated with 1-year mortality of HCC patients after TACE. Moreover, our study showed that the HCC patients with H-VFA had significantly worse overall survival after TACE than those with L-VFA. It 
was considered that visceral fat tissue is the metabolically active component, whereas subcutaneous and intermuscular fat tissue is the inactive component [17]. Our findings suggest that the distribution of fat tissue is a major determinant of the prognosis of HCC patients after TACE.

The extent of visceral fat has been reported to differ between males and females [32]. Indeed, in the present study, visceral fat area differed between males (median, $\left.97.2 \mathrm{~cm}^{2}\right)$ and females (median, $\left.62.6 \mathrm{~cm}^{2}\right)(p=0.001$ ). Therefore, the optimal cut-off values for visceral fat area were analyzed in males and females separately. These values of our study were lower than those of the previous studies for metabolic syndrome in the Japanese population [33]. This might be due to the fact that our study sample was biased towards older patients, although the previous study sample was limited to middle-aged patients. Indeed, Hayashi et al. have reported that the visceral fat area differed by generation [34]. Moreover, the NAFLD patients in our study numbered only 4 (4.2\%) out of 96 patients.

Visceral fat tissue is no longer classified as an inert site solely for energy homeostasis. In fact, it is now characterized as the largest multifunctional endocrine organ that participates in the regulation of hormonal balance of the body in patients with not only NAFLD, but also viral cirrhosis [35]-[37]. Our study showed that high visceral fat was associated with poor HbA1c. Previous studies showed that visceral fat has been reported to be associated with negative effects against glucose metabolism [38]. Visceral fat tissue controls the function of the pancreas by secreting adipokines [39]. The disturbance of adipokine balance by excessive visceral fat tissue could play a role in worsening glucose metabolism. Our study also showed that high visceral fat was associated with the TNM stage of HCC. A situation of worse glucose metabolism promotes cellular growth and increases free insulin-like growth factor levels, which plays an important role in tumor growth and differentiation [40] [41]. Visceral fat accumulation may be involved in both tumor initiation and promotion or progression steps through these mechanisms.

In our study, sarcopenia as defined previously [24] [25] was not associated with 1-year mortality and overall survival after TACE. In general, sarcopenia associated with mortality has progressive functional impairment, mainly Child's grade C. For example, these patients were waiting for liver transplantation [42] [43]. In our study, all of the HCC patients retained their liver function, namely, Child's grade A and B, not grade C. Sarcopenia associated with mortality has also been related to low quality of life (QOL) [44]. In our study, all patients had PS 0 or 1 and retained good QOL. Moreover, sarcopenia associated with mortality was reported to increase the risk of sepsis-related death in cirrhotic patients, suggesting impaired immunity [45]. In our study, liver failure and HCC progression after TACE, but not sepsis, were the causes of death in all of the patients. Therefore, it is considered that sarcopenia would not be necessarily related to mortality in cirrhotic patients.

Many hepatologists have needed new prognostic models after HCC treatment [46]. We considered that the prognosis of patients after TACE would be better evaluated by visceral fat area. Using the value of visceral fat area, patients who have a potentially high risk of 1-year mortality after TACE could be detected. This value is not available for the selection of HCC patients for whom TACE would not be recommended. However, for early detection and prevention of 1-year mortality risk after TACE, this value is available. To prevent 1-year mortality risk after TACE, nutritional support is thought to be necessary in cirrhotic patients with advanced HCC undergoing TACE. L-carnitine is an essential cofactor in the transport of long-chain fatty acids, such as acylcarnitine esters, across the inner mitochondrial membrane for subsequent fat degradation [47]. Previous studies have shown that visceral steatosis was found in the loss of L-carnitine transport activity [48]. In addition, the administration of L-carnitine reduced the accumulation of visceral fat mass [49]. Nutritional supplementation with oral L-carnitine might be beneficial in reducing visceral fat, and in improving the mortality rate in HCC patients undergoing TACE. Our predictive model was established by the value of visceral fat area without a secondary effect by L-carnitine supplementation. Therefore, using the value of visceral fat area, appropriate nutritional support could be administered to HCC patients undergoing TACE while avoiding the provision of unnecessary nutritional support to patients with a low risk of 1-year mortality after TACE.

Our study has several limitations. First, it is a single institutional study with a relatively small sample size of HCC patients undergoing TACE. Second, the study is observational, so we could not infer causality between visceral fat accumulation and mortality. Third, the cut-off value of visceral fat area might differ in patients with liver disease. Finally, we have not yet validated predictive factors associated with 1-year mortality after TACE. At present, we are recruiting a different cohort to validate its ability, which we will report on in the near future.

\section{Conclusion}

In conclusion, our study showed that H-VFA was a prognostic factor associated with survival in HCC patients 
undergoing TACE. Using the value of visceral fat area, 1-year mortality risk after TACE would be better estimated before the day TACE was performed.

\section{Acknowledgements}

We thank the nurses and our colleagues in the Department of Gastroenterology and the Gastroenterology Ward at Kobe University Hospital for their cooperation. This work was supported by a Grant-in-Aid for Young Scientists (B) [M.S.], and was also supported by grants for the Global COE Program, Global Center of Excellence for Education and Research on Signal Transduction Medicine in the Coming Generation from the Ministry of Education, Culture, Sports, Science, and Technology of Japan [M.Y. and T.A.].

\section{Conflict of Interest}

We, the authors, declare that we have no conflicts of interest.

\section{References}

[1] Bronowicki, J.P., Vetter, D., Dumas, F., et al. (1994) Transcatheter Oily Chemoembolization for Hepatocellular Carcinoma. A 4-Year Study of 127 French Patients. Cancer, 74, 16-24. http://dx.doi.org/10.1002/1097-0142(19940701)74:1<16::AID-CNCR2820740105>3.0.CO;2-V

[2] Vetter, D., Wenger, J.J., Bergier, J.M., et al. (1991) Transcatheter Oily Chemoembolization in the Management of Advanced Hepatocellular Carcinoma in Cirrhosis: Results of a Western Comparative Study in 60 Patients. Hepatology, 13, 427-433. http://dx.doi.org/10.1002/hep.1840130308

[3] Mondazzi, L., Bottelli, R., Brambilla, G., et al. (1994) Transcatheter Oily Chemoembolization for the Treatment of Hepatocellular Carcinoma: A Multivariate Analysis of Prognostic Factors. Hepatology, 19, 1115-1123. http://dx.doi.org/10.1002/hep.1840190508

[4] Lo, C.M., Ngan, H., Tso, W.K., et al. (2002) Randomized Controlled Trial of Transarterial Lipiodol Chemoembolization for Unresectable Hepatocellular Carcinoma. Hepatology, 35, 1164-1171. http://dx.doi.org/10.1053/jhep.2002.33156

[5] Llovet, J.M., Real, M.I., Montaňa, X., et al. (2002) Arterial Embolisation or Chemoembolisation versus Symptomatic Treatment in Patients with Unresectable Hepatocellular Carcinoma: A Randomised Controlled Trial. The Lancet, 359, 1734-1739. http://dx.doi.org/10.1016/S0140-6736(02)08649-X

[6] Llovet, J.M. and Bruix, J. (2003) Systematic Review of Randomized Trials for Unresectable Hepatocellular Carcinoma: Chemoembolization Improves Survival. Hepatology, 37, 429-442. http://dx.doi.org/10.1053/jhep.2003.50047

[7] Bismuth, H., Morino, M., Sherlock, D., et al. (1992) Primary Treatment of Hepatocellular Carcinoma by Arterial Chemoembolization. The American Journal of Surgery, 163, 387-394. http://dx.doi.org/10.1016/0002-9610(92)90039-T

[8] Yamada, R., Kishi, K., Sonomura, T., et al. (1990) Transcatheter Arterial Embolization in Unresectable Hepatocellular Carcinoma. CardioVascular and Interventional Radiology, 13, 135-139. http://dx.doi.org/10.1007/BF02575464

[9] Stuart, K., Stokes, K., Jenkins, R., et al. (1993) Treatment of Hepatocellular Carcinoma Using Doxorubicin/Ethiodized Oil/Gelatin Powder Chemoembolization. Cancer, 72, 3202-3209. http://dx.doi.org/10.1002/1097-0142(19931201)72:11<3202::AID-CNCR2820721112>3.0.CO;2-4

[10] Bruix, J. and Sherman, R. (2005) Management of Hepatocellular Carcinoma. Hepatology, 42, 1208-1236. http://dx.doi.org/10.1002/hep.20933

[11] Pugh, R.N., Murray-Lyon, I.M., Dawson, J.L., et al. (1973) Transection of the Oesophagus for Bleeding Oesophageal Varices. British Journal of Surgery, 60, 646-649. http://dx.doi.org/10.1002/bjs.1800600817

[12] Kamath, P.S., Wiesner, R.H., Malinchoc, M., et al. (2001) A Model to Predict Survival in Patients with End-Stage Liver Disease. Hepatology, 33, 464-470. http://dx.doi.org/10.1053/jhep.2001.22172

[13] Vauthey, J.N., Lauwers, G.Y., Esnaola, N.F., et al. (2002) Simplified Staging for Hepatocellular Carcinoma. Journal of Clinical Oncology, 20, 1527-1536. http://dx.doi.org/10.1200/JCO.20.6.1527

[14] Calle, E.E., Rodriguez, C., Walker-Thurmond, K., et al. (2003) Overweight, Obesity, and Mortality from Cancer in a Prospectively Studied Cohort of U.S. Adults. The New England Journal of Medicine, 348, 1625-1638. http://dx.doi.org/10.1056/NEJMoa021423

[15] Ohki, T., Tateishi, R., Sato, T., et al. (2008) Obesity Is an Independent Risk Factor for Hepatocellular Carcinoma Development in Chronic Hepatitis C Patients. Clinical Gastroenterology and Hepatology, 6, 459-464.

http://dx.doi.org/10.1016/j.cgh.2008.02.012 
[16] Saito, M., Seo, Y., Yano, Y., et al. (2013) Reduction in Non-Protein Respiratory Quotient Is Related to Overall Survival after Hepatocellular Carcinoma Treatment. PLOS ONE, 8, e55441. http://dx.doi.org/10.1371/journal.pone.0055441

[17] Mathur, A., Hernandez, J., Shaheen, F., et al. (2011) Preoperative Computed Tomography Measurements of Pancreatic Steatosis and Visceral Fat: Prognostic Markers for Dissemination and Lethality of Pancreatic Adenocarcinoma. HPB (Oxford), 13, 404-410. http://dx.doi.org/10.1111/j.1477-2574.2011.00304.x

[18] House, M.G., Fong, Y., Arnaoutakis, D.J., et al. (2008) Preoperative Predictors for Complications after Pancreaticoduodenectomy: Impact of BMI and Body Fat Distribution. Journal of Gastrointestinal Surgery, 12, 270-278. http://dx.doi.org/10.1007/s11605-007-0421-7

[19] Kudo, M. and Okanoue, T. (2007) Management of Hepatocellular Carcinoma in Japan: Consensus-Based Clinical Practice Manual Proposed by the Japan Society of Hepatology. Oncology, 72, 2-15. http://dx.doi.org/10.1159/000111702

[20] Mitsiopoulos, N., Baumgartner, R.N., Heymsfield, S.B., et al. (1998) Cadaver Validation of Skeletal Muscle Measurement by Magnetic Resonance Imaging and Computerized Tomography. Journal of Applied Physiology, 85, 115122.

[21] Kvist, H., Sjöström, L. and Tylén, U. (1986) Adipose Tissue Volume Determinations in Women by Computed Tomography: Technical Considerations. International Journal of Obesity, 10, 53-67.

[22] Vehmas, T., Kairemo, K.J. and Taavitsainen, M.J. (1996) Measuring Visceral Adipose Tissue Content from Contrast Enhanced Computed Tomography. International Journal of Obesity and Related Metabolic Disorders, 20, 570-573.

[23] Mourtzakis, M., Prado, C.M., Lieffers, J.R., et al. (2008) A Practical and Precise Approach to Quantification of Body Composition in Cancer Patients Using Computed Tomography Images Acquired during Routine Care. Applied Physiology, Nutrition, and Metabolism, 33, 997-1006. http://dx.doi.org/10.1139/H08-075

[24] Prado, C.M., Lieffers, J.R., McCargar, L.J., et al. (2008) Prevalence and Clinical Implications of Sarcopenic Obesity in Patients with Solid Tumours of the Respiratory and Gastrointestinal Tracts: A Population-Based Study. The Lancet Oncology, 9, 629-635. http://dx.doi.org/10.1016/S1470-2045(08)70153-0

[25] Montano-Loza, A.J., Meza-Junco, J., Prado, C.M., et al. (2012) Muscle Wasting Is Associated with Mortality in Patients with Cirrhosis. Clinical Gastroenterology and Hepatology, 10, 166-173. http://dx.doi.org/10.1016/j.cgh.2011.08.028

[26] Kashihara, H., Lee, J.S., Kawakubo, K., et al. (2009) Criteria of Waist Circumference According to Computed Tomography-Measured Visceral Fat Area and the Clustering of Cardiovascular Risk Factors. Circulation Journal, 73, 18811886. http://dx.doi.org/10.1253/circj.CJ-09-0183

[27] Starley, B.Q., Calcagno, C.J. and Harrison, S.A. (2010) Nonalcoholic Fatty Liver Disease and Hepatocellular Carcinoma: A Weighty Connection. Hepatology, 51, 1820-1832. http://dx.doi.org/10.1002/hep.23594

[28] Kopelman, P.G. (2000) Obesity as a Medical Problem. Nature, 404, 635-643.

[29] Regimbeau, J.M., Colombat, M., Mognol, P., et al. (2004) Obesity and Diabetes as a Risk Factor for Hepatocellular Carcinoma. Liver Transplantation, 10, S69-S73. http://dx.doi.org/10.1002/lt.20033

[30] Gonzalez, L., Blanc, J.F., Sa Cunha, A., et al. (2004) Obesity as a Risk Factor for Hepatocellular Carcinoma in a Noncirrhotic Patient. Seminars in Liver Disease, 24, 415-419. http://dx.doi.org/10.1055/s-2004-860870

[31] Nair, S., Mason, A., Eason, J., et al. (2002) Is Obesity an Independent Risk Factor for Hepatocellular Carcinoma in Cirrhosis? Hepatology, 36, 150-155. http://dx.doi.org/10.1053/jhep.2002.33713

[32] Fox, C.S., Massaro, J.M., Hoffmann, U., et al. (2007) Abdominal Visceral and Subcutaneous Adipose Tissue Compartments: Association with Metabolic Risk Factors in the Framingham Heart Study. Circulation, 116, 39-48. http://dx.doi.org/10.1161/CIRCULATIONAHA.106.675355

[33] Oka, R., Kobayashi, J., Yagi, K., et al. (2008) Reassessment of the Cutoff Values of Waist Circumference and Visceral Fat Area for Identifying Japanese Subjects at Risk for the Metabolic Syndrome. Diabetes Research and Clinical Practice, 79, 474-481. http://dx.doi.org/10.1016/j.diabres.2007.10.016

[34] Hayashi, T., Boyko, E.J., McNeely, M.J., et al. (2007) Minimum Waist and Visceral Fat Values for Identifying Japanese Americans at Risk for the Metabolic Syndrome. Diabetes Care, 30, 120-127. http://dx.doi.org/10.2337/dc06-0739

[35] Eguchi, Y., Mizuta, T., Ishibashi, E., et al. (2009) Hepatitis C Virus Infection Enhances Insulin Resistance Induced by Visceral Fat Accumulation. Liver International, 29, 213-220. http://dx.doi.org/10.1111/j.1478-3231.2008.01853.x

[36] González-Reimers, E., Castellano-Higuera, A., Alemán-Valls, R., et al. (2009) Relation between Body Fat and Liver Fat Accumulation and Cytokine Pattern in Non-Alcoholic Patients with Chronic HCV Infection. Annals of Nutrition and Metabolism, 55, 351-357. http://dx.doi.org/10.1159/000252351

[37] Ahima, R.S. (2006) Adipose Tissue as an Endocrine Organ. Obesity, 14, 242S-249S. 
http://dx.doi.org/10.1038/oby.2006.317

[38] Vongsuvanh, R., George, J., Qiao, L., et al. (2013) Visceral Adiposity in Gastrointestinal and Hepatic Carcinogenesis. Cancer Letters, 330, 1-10. http://dx.doi.org/10.1016/j.canlet.2012.11.038

[39] Arano, T., Nakagawa, H., Tateishi, R., et al. (2011) Serum Level of Adiponectin and the Risk of Liver Cancer Development in Chronic Hepatitis C Patients. International Journal of Cancer, 129, 2226-2235. http://dx.doi.org/10.1002/ijc.25861

[40] Alexia, C., Fallot, G., Lasfer, M., et al. (2004) An Evaluation of the Role of Insulin-Like Growth Factors (IGF) and of Type-I IGF Receptor Signalling in Hepatocarcinogenesis and in the Resistance of Hepatocarcinoma Cells against Drug-Induced Apoptosis. Biochemical Pharmacology, 68, 1003-1015. http://dx.doi.org/10.1016/j.bcp.2004.05.029

[41] Giovannucci, E. and Michaud, D. (2007) The Role of Obesity and Related Metabolic Disturbances in Cancers of the Colon, Prostate, and Pancreas. Gastroenterology, 132, 2208-2225. http://dx.doi.org/10.1053/j.gastro.2007.03.050

[42] Kaido, T., Ogawa, K., Fujimoto, Y., et al. (2013) Impact of Sarcopenia on Survival in Patients Undergoing Living Donor Liver Transplantation. American Journal of Transplantation, 13, 1549-1556. http://dx.doi.org/10.1111/ajt.12221

[43] Masuda, T., Shirabe, K., Ikegami, T., et al. (2014) Sarcopenia Is a Prognostic Factor in Living Donor Liver Transplantation. Liver Transplantation, 20, 401-407. http://dx.doi.org/10.1002/lt.23811

[44] Fearon, K.C. (2011) Cancer Cachexia and Fat-Muscle Physiology. The New England Journal of Medicine, 365, 565567. http://dx.doi.org/10.1056/NEJMcibr1106880

[45] Merli, M., Lucidi, C., Giannelli, V., et al. (2010) Cirrhotic Patients Are at Risk for Health Care-Associated Bacterial Infections. Clinical Gastroenterology and Hepatology, 8, 979-985. http://dx.doi.org/10.1016/j.cgh.2010.06.024

[46] Wang, Y., Chen, Y., Ge, N., et al. (2012) Prognostic Significance of Alpha-Fetoprotein Status in the Outcome of Hepatocellular Carcinoma after Treatment of Transarterial Chemoembolization. Annals of Surgical Oncology, 19, 35403546. http://dx.doi.org/10.1245/s10434-012-2368-5

[47] Tein, I., Bukovac, S.W. and Xie, Z.W. (1996) Characterization of the Human Plasmalemmal Carnitine Transporter in Cultured Skin Fibroblasts. Archives of Biochemistry and Biophysics, 329, 145-155. http://dx.doi.org/10.1006/abbi.1996.0203

[48] Yokogawa, K., Yonekawa, M., Tamai, I., et al. (1999) Loss of Wild-Type Carrier-Mediated L-Carnitine Transport Activity in Hepatocytes of Juvenile Visceral Steatosis Mice. Hepatology, 30, 997-1001. http://dx.doi.org/10.1002/hep.510300423

[49] Kim, Y.J., Kim, K.Y., Kim, M.S., et al. (2008) A Mixture of the Aqueous Extract of Garcinia cambogia, Soy Peptide and L: -Carnitine Reduces the Accumulation of Visceral Fat Mass in Rats Rendered Obese by a High Fat Diet. Genes \& Nutrition, 2, 353-358. http://dx.doi.org/10.1007/s12263-007-0070-1 